\title{
The Impact of Joint Replacement on Driver Function and Safety
}

\author{
Stephanie J. Muh ${ }^{1 *}$, Yousef Shishani ${ }^{1}$, Jonathan Streit ${ }^{1}$, Carissa Ann Lucas ${ }^{2}$, Vinod Sahgal ${ }^{3}$, \\ Matthew Kraay ${ }^{3}$, Reuben Gobezie ${ }^{4}$
}

${ }^{1}$ Case Shoulder and Elbow Service, Case Western Reserve University School of Medicine, University Hospitals of Cleveland, Cleveland, USA; ${ }^{2}$ Department of Hematology/Oncology - Solid Tumor Department, The Cleveland Clinic, Cleveland, USA; ${ }^{3}$ Department of Orthopaedics, Case Western Reserve University School of Medicine, University Hospitals of Cleveland, Cleveland, USA; ${ }^{4} \mathrm{Cleveland}$ Shoulder Institute, University Hospitals of Cleveland, Cleveland, USA.

Email: *stephanie.muh@gmail.com

Received June $24^{\text {th }}, 2012$; revised July $28^{\text {th }}, 2012$; accepted August $10^{\text {th }}, 2012$

\begin{abstract}
Background: The timing of return-to-driving following total joint replacement has not been well-defined. The primary aim is to study the impact of joint replacement on driver safety. A secondary aim is to investigate the possible predictors for increased car accidents in patients undergoing total joint replacement surgery. This data will provide the background to support further prospective studies on the relationship of driver safety to joint replacement surgery. Methods: A retrospective analysis of driver safety in the postoperative period was performed by analyzing the self-reporting of 485 patients who had undergone a Total Hip Arthroplasty $(n=196)$, Total Knee Arthroplasty $(n=258)$ or Total Shoulder Arthroplasty $(\mathrm{n}=31)$. The mean age was $70(28$ - 88) with 164 male $(34 \%)$ and 319 female $(66 \%)$. Patients were mailed a questionnaire and the responses were analyzed to determine what factors affect driving after joint replacement. Results: Overall, increased patient age and gender were associated with increased accidents following surgery. Our subgroup analysis demonstrated that in patients undergoing TSA, increased patient age was associated with increased accidents. TKA patients showed that older patient age predicted increased accidents following surgery. Conclusion: While age and gender are correlated with increased accidents after joint replacement in general, no factors specific to joint replacement surgery are related to increased incidence of accidents.
\end{abstract}

Keywords: Total Hip Arthroplasty; Total Knee Arthroplasty; Total Shoulder Arthroplasty; Driving; Driver Safety; Traffic Accidents

\section{Background}

Long term projections of joint replacement show a continued increase in demand for total shoulder, hip and knee arthroplasties [1,2]. After a joint replacement, a patient's everyday life is profoundly affected due to the inability to drive postoperatively [3]. Many patients are eager to resume driving as early as possible and it is often up to the orthopaedic surgeon to advise their patient on when to drive. With little evidence available and limited guidelines in the literature, the orthopaedic surgeon has the difficult task of making a recommendation in regards to a patient's ability to return to driving after surgery. Most surgeons do not know when patients return to driving regardless of their recommendations. It is possible that multiple factors, such as patient confidence, prior injury, and other socioeconomic issues impact a patient's decision to resume driving. To better understand our pa-

*Corresponding author. tients' expectations and habits in the postoperative period, we conducted a survey to see how soon they began driving following total hip arthroplasty (THA), total knee arthroplasty (TKA) and total shoulder arthroplasty (TSA). Additionally, we asked the patients to rate the impact of surgery on their confidence and perceived ability to drive.

\section{Materials and Methods}

Our study received Institutional Review Board approval, and all individuals consented to participate. All patients undergoing primary THA, TKA or TSA for a diagnosis of osteoarthritis or fracture between the dates of January 1, 1997 and December 31, 2005 were identified by using our institution's joint registry database. Inclusion criteria for involvement in the study were age greater than 18 years and a diagnosis of osteoarthritis or fracture unresponsive to non-operative management as the surgical indication. Exclusion criteria were age less than 18 years, 
inability to read English, prior surgery, revision arthroplasty, and any post-operative complications.

All procedures were performed by three fellowshiptrained total joint replacement surgeons. A standard postoperative course was followed for each patient, which consisted of inpatient hospitalization until adequate pain control had been achieved, and regular follow-up visits beginning two weeks after surgery. Although rehabilitation exercises were prescribed, no specific guidelines were given to patients with regard to the timing of return to driving following surgery, other than complete cessation of narcotics. Patients were encouraged to practice in a parking lot prior to driving on roads, and to use good judgment in assessing their ability to drive safely.

A total of (1025) patients met the criteria for inclusion in this study. Of those, there were (424) THA patients, (514) TKA patients and (87) TSA patients. These patients were mailed a questionnaire as well as a detailed letter from the principal investigator (RG) inviting them to participate. The questionnaire asked patients to recall how soon following surgery they began driving an automatic transmission, as well as how confident they were in their driving abilities after surgery. Additionally, they were asked to rate the impact joint replacement surgery had on their ability to drive. Patients were instructed to return the completed questionnaires and were then deidentified and collected in a database for review. Four hundred and eighty-five (485) patients agreed to participate in the study. Of these, (196) were THA patients, (258) were TKA patients and (31) were TSA patients. The questionnaire was sent between 24 and 144 months from the time of surgery. A logistics model was used to predict factors that affected the number of accidents a patient would have after any type of arthroplasty. Significance was set at $\mathrm{p}<0.05$.

\section{Results}

The average age of patients in the study was $70.0+/-9.0$ years, with the average total hip arthroplasty (THA) being $70.5+/-10.1$ years, total knee arthroplasty (TKA) being $70.1+/-7.6$ years and total shoulder arthoplasty (TSA) being $66.3+/-11.5$ years (Table 1).

In general, patients were very confident in their driving abilities following arthroplasty of all three joints. On a scale of 1 to 5 , with 5 being most confident and 1 being not confident, patients reported a confidence of $4.9+/-$ 0.4 . TSA patients reported a mean of $4.90+/-0.3$, patients post THA reported a mean $4.89+/-0.45$, and TKA patients reported a mean of $4.91+/-0.38$ (Table 2). Furthermore, $100 \%$ of patients after TSA, $98 \%$ of patients after THA, and $98 \%$ of patients following TKA reported no change or improvement in driving ability after total joint arthroplasty (Table 3).
Most patients following total joint replacement returned to driving in less than 6 months (Table 4). In general, $24 \%(n=113)$ of patients started driving in less than 1 month after surgery. Within 3 months, $96 \%(n=463)$ of patients were driving and $98 \%(\mathrm{n}=472)$ were driving within 6 months. There were a total of 49 patients who were involved in accidents after having a joint replacement and $96 \%$ (47/49) were within the first six months post-operatively (Table 5). $38.7 \%(n=12)$ of the patients with a total shoulder replacement started driving within 1 month, 93.5\% $(\mathrm{n}=29)$ within 3 months, and 96.7\% $(\mathrm{n}=$ 30) within 6 months. There were 2 accidents in the TSA group with 1 in a patient who returned to driving within 1 month of surgery $(8.33 \%)$, and another who returned in 1 3 months (5.89\%). The TKA and THA groups resumed driving slower than TSA patients, with only $25.2 \%$ and $18.6 \%$ respectively, resuming driving within 1 month of their surgery. However, following TKA, $96.5 \%$ of patients resumed driving within 3 months, and $97.3 \%$ had resumed driving within 6 months. In this group there were a total of 25 accidents with 10 occurring in patients who returned to driving within 1 month (15.4\%), 15 in

Table 1. Patient demographics.

\begin{tabular}{cccccc}
\hline & Age & Significance & Male & Female & Significance \\
\hline TSA & $\begin{array}{c}66.3 \\
(+/-11.5)\end{array}$ & $\mathrm{p}=0.03^{*}$ & $17(55 \%)$ & $14(45 \%)$ & $\mathrm{p}=0.87$ \\
& & & & \\
TKA & $\begin{array}{c}70.1 \\
(+/-7.6)\end{array}$ & $\mathrm{p}=0.06$ & $73(28 \%)$ & $185(72 \%)$ & $\mathrm{p}=0.01^{*}$ \\
& $\begin{array}{c}70.5 \\
\text { THA } \\
(+/-10.1)\end{array}$ & $\mathrm{p}=0.43$ & $74(38 \%)$ & $120(62 \%)$ & $\mathrm{p}=0.35$ \\
& $\begin{array}{c}70.0 \\
\text { Total } \\
(+/-9.0)\end{array}$ & $\mathrm{p}=0.016^{*}$ & $164(34 \%)$ & $319(66 \%)$ & $\mathrm{p}=0.039^{*}$ \\
\hline
\end{tabular}

${ }^{*} \mathrm{p}<0.05=$ statistically significant.

Table 2. Confidence in driving ability following total joint replacement.

\begin{tabular}{cccc}
\hline TSA & TKA & THA & Total \\
\hline $4.90+/-0.30$ & $4.91+/-0.38$ & $4.89+/-0.45$ & $4.90+/-0.40$ \\
\hline
\end{tabular}

Table 3. Self perception of surgery's impact on driving ability.

\begin{tabular}{lcccc}
\hline & $\begin{array}{c}\text { Improved } \\
(\%)\end{array}$ & $\begin{array}{c}\text { No change } \\
(\%)\end{array}$ & $\begin{array}{c}\text { Worsened } \\
(\%)\end{array}$ & $\begin{array}{c}\text { Missed } \\
(\%)\end{array}$ \\
\hline TSA $(\mathrm{n}=31)$ & $9(29 \%)$ & $22(71 \%)$ & $0(0 \%)$ & $0(0 \%)$ \\
TKA $(\mathrm{n}=258)$ & $65(25 \%)$ & $188(73 \%)$ & $1(0.4 \%)$ & $4(1.6 \%)$ \\
THA (n=194) & $48(24.7 \%)$ & $143(73.7 \%)$ & $3(1.6 \%)$ & $0(0 \%)$ \\
Total $(\mathrm{n}=483)$ & $122(25.3 \%)$ & $353(73.1 \%)$ & $4(0.8 \%)$ & $4(0.8 \%)$ \\
\hline
\end{tabular}


Table 4. Number of months after surgery when patient returned to driving.

\begin{tabular}{ccccccccc}
\hline & $<1 \mathrm{mo}(\%)$ & $1-3 \mathrm{mo}(\%)$ & $<6 \mathrm{mo}(\%)$ & $<1 \mathrm{yr}(\%)$ & $>1 \mathrm{yr}(\%)$ & Never (\%) & N/a (\%) & Total \\
\hline TSA & $12(38.71 \%)$ & $17(54.8 \%)$ & $1(3.23 \%)$ & $0(0 \%)$ & $0(0 \%)$ & $0(0 \%)$ & $1(3.23 \%)$ & 31 \\
TKA & $65(25.19 \%)$ & $184(71.32 \%)$ & $2(0.77 \%)$ & $1(0.39 \%)$ & $1(0.39 \%)$ & $0(0 \%)$ & $5(1.95 \%)$ & 258 \\
THA & $36(18.6 \%)$ & $149(76.8 \%)$ & $6(3.09 \%)$ & $0(0 \%)$ & $1(0.52 \%)$ & $2(1.03 \%)$ & $2(1.03 \%)$ & 196 \\
Total & $113(23.89 \%)$ & $350(72.5 \%)$ & $9(1.86 \%)$ & $1(0.21 \%)$ & $2(0.41 \%)$ & $2(0.41 \%)$ & $8(1.66 \%)$ & 485 \\
\hline
\end{tabular}

Table 5. Post-operative accidents compared to time return to driving.

\begin{tabular}{|c|c|c|c|c|c|c|}
\hline & $\begin{array}{l}\text { "Patients returning to } \\
\text { drive }(<1 \mathrm{mo})\end{array}$ & $\begin{array}{l}\text { Accidents } \\
(\%)\end{array}$ & $\begin{array}{c}\text { "Patients returning to drive } \\
\qquad(1-3 \mathrm{mo})\end{array}$ & $\begin{array}{l}\text { Accidents } \\
(\%)\end{array}$ & $\begin{array}{l}\text { "Patients returning to } \\
\text { drive }(<6 \mathrm{mo})\end{array}$ & $\begin{array}{l}\text { Accidents } \\
(\%)\end{array}$ \\
\hline TSA & 12 & $1(8.33 \%)$ & 17 & $1(5.89 \%)$ & 1 & $0(0 \%)$ \\
\hline TKA $^{*}$ & 65 & $10(15.38 \%)$ & 184 & $15(8.15 \%)$ & 2 & $0(0 \%)$ \\
\hline THA & 36 & $1(2.78 \%)$ & 149 & $19(12.75 \%)$ & 6 & $2(33.33 \%)$ \\
\hline Total & 113 & $12(10.60 \%)$ & 350 & $35(10 \%)$ & 9 & $2(22.2 \%)$ \\
\hline
\end{tabular}

${ }^{*} 1$ patient did not respond on time return to driving.

patients who returned to driving within 3 months $(8.15 \%)$. One patient did not respond when they returned driving. Similarly, after THA, $95.4 \%$ of patients resumed driving within 3 months and $98.5 \%$ resumed driving within 6 months of their surgery. There was a total of 22 accidents with 1 accident within 1 month $(2.78 \%), 19$ within 3 months (12.75\%) and 2 within 6 months (33.33\%).

Overall, increasing age $(\mathrm{p}=0.016)$ and gender $(\mathrm{p}=$ $0.039)$ were significantly correlated with increased accidents post-arthroplasty. There was no correlation between other investigated variables (time return to driving or perceived patient opinion on how joint arthroplasty affected their driving ability) and incidence of postoperative accidents. An odds ratio demonstrates that women have 1.5 increased odds of having accidents postoperatively than men. For patients who had total shoulder arthroplasty, patients had an increased incidence of accidents postoperatively with increasing age $(p=0.03)$. For patients who had total knee arthroplasty, gender $(p=0.01)$ was associated with more accidents following surgery. After TKA, females had 2.12 increased odds compared to males. However, for total hip arthroplasty, no factors were associated with increased accidents after surgery.

\section{Discussion}

The recommended time for a patient to return to driving after total joint arthroplasty is not clearly defined in the literature. One factor thought to predict driving ability is reaction time, or the time it takes to react to a stimulus and move from the accelerator to the brake. The literature quotes anywhere from 4 - 6 weeks to up to 8 months following hip arthroplasty [4,5] and 10 days to 8 weeks for total knee arthroplasty [6-9]. To our knowledge, there is no literature in regards to total shoulder arthroplasty and when patients can safely return to driving.

Our study suggests that the factors that determine post-operative driving ability vary based on the specific joint replaced. Chen et al. [3] conducted a survey of 70 patients who refrained from driving following fracture and found that the inability to drive presented a major difficulty to $42 \%$ of respondents, with $31 \%$ reporting a minor difficulty. Additionally, the inability to drive caused major financial hardship in $26 \%$ of respondents. Because inability to drive causes such a great inconvenience, many patients are eager to return to normal driving habits following surgery. Indeed, Chen et al. [3] found that many patients did not consult with their doctors before resuming driving, and that many started driving while still taking opioid pain medications. The issue of readiness to drive following fractures of the extremities was also studied by Rees and Sharp [10], who found a large variability in response among orthopaedic surgeons presented with scenarios involving upper extremity deficits, with majority agreement in only $43 \%$ of different presented scenerios. Also, in a study of orthopaedic surgeon activity recommendations following trauma, Chen et al. found a high variability in response to the question of readiness to drive following shoulder fracture [3]; respondents gave recommendations ranging from 0 to 12 weeks, and $76 \%$ of respondents used no consistent guidelines when advising patients about a safe return to driving. 
In our study, we found that patient's age and gender were found to be important in increased incidence of accidents post-operatively. Overall, older patients were at higher risk of being involved in accidents post-operatively. Additionally, females had higher odds of postoperative accidents compared to men. In the TSA group, only increased patient age was associated with increased accidents following surgery, and the specific time to returning to drive did not predict accidents following surgery. This association appears intuitive, as patients age, they can develop increasing debilitating conditions that can place them at-risk for collisions. Despite this, $94 \%$ of patients returned to driving within 3 months. This issue is especially important because a large proportion of patients undergoing TSA are over the age of 65 . Studies by the National Highway Traffic Safety Administration have shown that drivers over age 65 have one of the highest rates of traffic accidents per mile driven [11]. While reaction time and judgment certainly play a large role in fatal accidents, upper extremity function is at least one of the factors which must also be considered as demonstrated by the fact that in $27 \%$ of fatal traffic accidents involving a driver over the age of 65 , the driver was turning left.

In our study the majority of our patients returned to driving within 6 months after joint arthroplasty. Current literature recommends patient return to driving $4-8$ weeks after total hip surgery $[4,5]$ and anywhere from 10 days to 8 weeks following TKA [6-9]. Even though our patients seemed to fall within these recommended guidelines, all recorded accidents occurred within 6 months. Therefore, other factors may play a role in contributing to accidents after surgery that were not investigated in this study. In separate prospective studies, Marques et al., found that brake response time improved at 30 days for patients undergoing right TKA and 10 days for left TKA. The authors concluded that right TKA patients should wait 30 days prior to return to driving automatic transmissions and left TKA patients may return in 10 days [7,9]. MacDonald et al. suggested that some patients can take up to 8 months to completely recover driving reaction time back to the pre-operative level [5]. Therefore, it is likely that patient safety in returning to drive after joint arthroplasty is dependent on multiple factors.

Limitations of our study relate to its retrospective design, the relative small number of patients, and the variety in the number of patients within each subgroup. Additionally, other joint arthroplasties were not investigated in this study. The increasing incidence of these arthroplasties (ankle, elbow, etc.) warrants future consideration. Despite its retrospective nature, our study reports on our patients opinions and beliefs about their driving ability and the objective driving outcomes.

\section{Conclusion}

Our results suggest that while age and gender plays a role in driver safety, there are many factors that determine post-operative driving ability. A significant number of patients return to driving within the first six months after joint replacement surgery. Early return to driving within the first six months may be related to an increased risk of accident. This should be considered when advising patients on when it is safe to return to drive after a joint replacement.

\section{REFERENCES}

[1] J. S. Day, E. Lau, K. L. Ong, G. R. Williams, M. L. Ramsey and S. M. Kurtz, "Prevalence and Projections of Total Shoulder and Elbow Arthroplasty in the United States to 2015," Journal of Shoulder and Elbow Surgery, Vol. 19, No. 8, 2010, pp. 1115-1120. doi:10.1016/j.jse.2010.02.009

[2] S. Kurtz, K. Ong, E. Lau, F. Mowat and M. Halpern, "Projections of Primary and Revision Hip and Knee Arthroplasty in the United States from 2005 to 2030," The Journal of Bone \& Joint Surgery of American, Vol. 89, No. 4, 2007, pp. 780-785. doi:10.2106/JBJS.F.00222

[3] V. Chen, A. T. Chacko, F. V. Costello, N. Desrosiers, P. Appleton and E. K. Rodriguez, "Driving after Musculoskeletal Injury. Addressing Patient and Surgeon Concerns in an Urban Orthopaedic Practice," The Journal of Bone \& Joint Surgery of American, Vol. 90, No. 12, 2008, pp. 2791-2797. doi:10.2106/JBJS.H.00431

[4] S. B. Ganz, A. Z. Levin, M. G. Peterson and C. S. Ranawat, "Improvement in Driving Reaction Time after Total Hip Arthroplasty," Clinical Orthopaedics and Related Research, Vol. 413, No. 413, 2003, pp. 192-200.

[5] W. MacDonald and J. W. Owen, "The Effect of Total Hip Replacement on Driving Reactions," Journal of Bone \& Joint Surgery of British, Vol. 70, No. 2, 1988, pp. 202-205.

[6] T. J. Spalding, J. Kiss, P. Kyberd, A. Turner-Smith and A. H. Simpson, "Driver Reaction Times after Total Knee Replacement," Journal of Bone \& Joint Surgery of British, Vol. 76, No. 5, 1994, pp. 754-756.

[7] C. J. Marques, J. Cabri, J. Barreiros, A. I. Carita, C. Friesecke and J. F. Loehr, "The Effects of Task Complexity on Brake Response Time before and after Primary Right Total Knee Arthroplasty," Archives of Physical Medicine and Rehabilitation, Vol. 89, No. 5, 2008, pp. 851-855. doi:10.1016/j.apmr.2007.10.025

[8] J. L. Pierson, D. R. Earles and K. Wood, "Brake Response Time after Total Knee Arthroplasty: When Is It Safe for Patients to Drive?" The Journal of Arthroplasty, Vol. 18, No. 7, 2003, pp. 840-843. doi:10.1016/S0883-5403(03)00326-7

[9] C. J. Marques, J. Barreiros, J. Cabri, A. I. Carita, C. Friesecke and J. F. Loehr, "Does the Brake Response Time of the Right Leg Change after Left Total Knee Arthroplasty? A Prospective Study," Knee, Vol. 15, No. 4, 2008, pp. 295-298. doi:10.1016/j.knee.2008.02.008 
[10] J. L. Rees and R. J. Sharp, "Safety to Drive after Common Limb Fractures," Injury, Vol. 33, No. 1, 2002, pp. 51-54. doi:10.1016/S0020-1383(00)00153-4
[11] National Center for Statistics and Analysis, "Traffic Safety Facts 1997: Older Population,” 2010. http://www-nrdnhtsa dot gov/Pubs/97OLDERPOP PDF 